\title{
Functional relation of land surface albedo with climatological variables: a review on remote sensing techniques and recent research developments
}

\begin{abstract}
Surface albedo has been documented as one of the Essential Climate Variables (ECV) of the Global Climate Observing System (GCOS) that governs the Earth's Radiation Budget. The availability of surface albedo data is necessary for a comprehensive environmental modelling study. Thus, both temporal and spatial scale issues need to be rectified. This study reports about the availability of surface albedo data through in-situ and remote sensing satellite observations. In this paper, we reviewed the existing models for surface albedo derivation and various initiatives taken by related environmental agencies in order to understand the issues of climate with respect to surface albedo. This investigation evaluated the major activities on albedo-related research specifically for the retrieval methods used to derive the albedo values. Two main existing albedo measurement methods are derived through in-situ measurement and remotely sensed observations. In-situ measurement supported with number of instruments and techniques such aspyrheliometers, pyranometers and Baseline Surface Radiation Network (BSRN) and remotely sensed observations using angularly integrated Bidirectional Reflectance Distribution Function (BRDF) by both geostationary and polar orbit satellites. The investigation results reveals that the temporal and spatial scaling is the major issues when the albedo values are needed for microclimatic study, i.e. high-resolution timeseries analyses and at heterogeneity and impervious surface. Thus, an improved technique of albedo retrieval at better spatial and temporal scale is required to fulfil the need for such kind of studies. Amongst many others, there are two downscaling methods that have been identified to be used in resolving the spatial scaling biased issues: Smoothing Filter-based Intensity Modulation (SFIM) and Pixel Block Intensity Modulation (PBIM). The temporal issues can be resolved using the multiple regression techniques of land surface temperature, selected air quality parameters, aerosol and daily skylight.
\end{abstract}

Keyword: Albedo; Modelling; Remote sensing; Geographical information system (GIS) 